\title{
ANEMIA IN PATIENTS WITH ACTIVE ULCERATIVE COLITIS
}

\author{
Antoni Stadnicki \\ MD, PhD, DSc, Profesor, Polonia Academy in Częstochowa, Poland \\ and Multidisciplinary Hospital, Jaworzno, Poland
}

\section{Anna Stadnicka}

Student, University of Economics and Innovation in Lublin, Poland e-mail: astadnicka007@gmail.com,orcid.org/0000-0001-5107-8551

\section{Józef Kurek}

$\mathrm{MD}, \mathrm{PhD}$, General, Endocrinology and Oncology Surgery, Multidisciplinary Hospital, Jaworzno, Poland

\section{Summary}

Iron deficiency anemia (IDA) is the most common complication in patients with IBD. In spite this, some investigators indicate that more than a third of IBD patients are not tested for anemia in the time of diagnosis. Aim. To provide a pathway to help achieve diagnosis a type of anemia in patients with moderate activity of ulcerative colitis (UC). Methods. Basic profile of iron turnover parameters, red blood cell indices, and C-reactive protein (CRP) in 20 anemic patients with mild/moderate active UC patients was described. Results. IDA was diagnosed in fifteen $75 \%$ of the patients, tree of which (15\%) had anemia of chronic disease (ACD), and $10 \%$ had mixed type of anemia (IDA/ACD). Conclusion. Ferritin, seems to play a central role in the definition and diagnosis of anemia in UC patients. Routine screening for anemia is important among patients with IBD. The cornerstone for the accurate management of anemia in UC patients lies in accurately diagnosing the type of anemia.

Keywords: anemia, iron deficiency anemia, IDA, ulcerative colitis, ferritin, CRP.

DOI: https://doi.org/10.23856/3877

\section{Introduction}

Ulcerative colitis (UC) together with Crohn's disease (CD) constitute inflammatory bowel diseases (IBD), idiopathic, chronic intestinal and systemic inflammation with spontaneously relapsing course. About $15-45 \%$ of the cases are associated with extraintestinal manifestations (Bernstein, 2019; Stadnicki, 1998). Anemia is the most common complication IBD. In retrospective cohort study anemia was present in $41.2 \%$ of patients at diagnosis $(47 \%$ and $33.8 \%$ of Chohn's disease and UC patients, respectively, being severe in 5.5\% (Lucendo, 2017). Similarly, in other study evaluating one hundred IBD patients (49 UC), 51 Crohn's disease). The prevalence of anemia was $41.2 \%$ for UC and $42.9 \%$ for CD (Oustamanolakis, 2011). The etiology of anemia among IBD patients is most commonly due to iron deficiency anemia (IDA) following or anemia of chronic disease (ACD) (Lucendo, 2017; Oustamanolakis, 2011; Testa, 2016). Anemia in IBD can also be the result of other factors: folic acid or vitamin B12 deficiency (megaloblastic anemia) in Chron's disease due to frequent involvement of the terminal ileum (Battat, 2014), drug induced folic acid deficiency and hemolysis.

In general, an occurrence of IDA is mainly the result of iron loss from chronic bleeding from the colon, which may lead to its exhaustion from the system. The deficiency usually develops slowly and in three stages of iron deficiency: First: latent deficiency; lowered systemic iron 
resources do not cause any clinical symptoms; the only parameter proving anemia is lowered ferritin concentration in the serum Second: hidden deficiency; exhausted iron reserves are shown in low ferritin concentration, low transferrin saturation and erythrocytes' protoporphirins growth; hemoglobin $(\mathrm{Hb})$ level is usually around the lower norm limit. Third: Hb significant decrease, low concentration of iron and ferritin, increased level of transferrin and decrease of transferrin saturation, as well as high values of erythrocytes' protoporphirins. After exhaustion of iron reserves the saturation of transferrin falls and simultaneously total iron binding capacity (TIBC) in the serum is growing. Erythrocytes are characterized by hipochromia and microcytosis, which is shown by decreased value of mean corpuscular volume of red blood cells (MCV), mean corpuscular hemoglobin $(\mathrm{MCH})$, and red cell distribution with (RDW), its growth may precede changes in MCV (Clarke, 2008). Anemia of chronic diseases (ACD), in which the pathogenic role is played by pro-inflammatory cytokines. It was found (Gomollon, 2009), that IL-1, and IL-6 are responsible for the development of ACD by inhibiting gene expression and erythropoietin secretion. An additional mechanism, for which IL-1 is responsible, is the synthesis of apoferritin as the acute phase protein, which binds the iron and then stores it in the form of ferritin, which effects in lowering of iron in the serum (Bertero, 1997). Anemia of chronic disease It is formed as a result of reduction of erythropoietin (EPO) synthesis, and reduction of the sensitivity of erythrocyte precursor cells to EPO (Weiss, 2005). IL-6 was recognized as the main mediator of ACD development, as stimulating the synthesis of hepcidin, a protein whose excess causes lowering of iron absorption from the duodenum (Mecklenburg, 2014). IL-6 is known as main stimulator of acute phase proteins, like C-reactive protein (CRP) in hepatocytes. Mutual dependence between IL-6 and CRP and their correlation with activity of the disease was observed in patients with Crohn's disease, the relation was less pronounced in case of UC (Vermeire, 2006).

Anemia is a relatively late stage manifestation of iron deficiency. Despite this, iron deficiency is generally not systematically screened for in IBD patients. It is clear from other disease areas including chronic heart failure (Anker, 2009) and chronic kidney disease (Shepshelovich, 2016). In this article a basic profile of iron turnover parameters in relation to $\mathrm{CRP}$, and red blood cell parameters in moderately active UC patients with is presented.

\section{Materials and Methods}

\section{Patients' characteristics.}

20 active UC patients were accepted for the examinations ( 9 women, 11 men, age average of 46 years) and 19 healthy volunteers ( 11 women and 8 men with average age of 46 years), constituting the control group.

UC was diagnosed on the basis of anamnesis data colonoscopy, histopathologic assessment of bowel samples and laboratory tests. UC patients with colitis fulminas and coexisting hepatitis, asthma and significant circulatory and respiratory failure were excluded from the studies. All UC patients were treated with 5-ASA preparations. Moreover, 8 patients with active stage of the disease were given moderately dose of steroid formulations, and additionally 5 patients were administered azatioprine. Among UC patients assessment of the disease's activeness was conducted on the basis of criteria developed by Truelove and Witts (Truelove, 1955). All patients were found to have moderately active stage of the disease, score between 3-8.

In UC patients and control patents blood for examinations was withdrawn from a vein in cubital fossa. Blood was rotated for 10 minutes with the speed of $3000 \mathrm{rpm}$ in order to extract the serum, which was kept in the temperature of $-70^{\circ} \mathrm{C}$. Before beginning to determine the parameters, the test tubes were warmed to room temperature. 


\section{Iron concentration}

Determining of serum iron concentration was carried out with photometrical method, with the use of "COBAS MIRA Plus" apparatus by Roche and the reagent Iron Sys1 by Roche. (reference values: $49-151$ for woman; 53-157 for man [ $\mu \mathrm{g} / \mathrm{dl}]$

\section{Ferritin level in serum}

Determining of serum ferritin level was carried out by immunoenzymatic method ELISA, in which two mouse monoclonal antibodies with high specifity were used. These antibodies are capable of finding most of ferritin's lysoforms. Horseradish peroxidase was used as the substrate in the reaction. All original reagents were parts of the kit "COBAS CORE Ferritin EIA" by F. Hoffman-La Roche, Basel, Switzerland. Measurements were made with COBAS CORE II apparatus by Roche (reference values: $13-150$ for woman; 30-400 for man $\mu \mathrm{g} / \mathrm{L}$ ).

\section{Evaluation of unsaturated iron binding capacity (UIBC), total iron-binding capacity (TIBC) and transterrin saturation}

Determining of and unsaturated iron binding capacity (UIBC) was carried out with photometrical method with the use of "COBAS MIRA Plus" apparatus by Roche and Iron Sys1 by Roche and UNIMATE 7 kits. Total Iron Binding Capacity (TIBC), value was calculated on the basis of the equation UIBC $=$ TIBC-iron concentration in serum. Reference values: TIBC 262-474 [ $\mu \mathrm{g} / \mathrm{dl}]$, UIBC112-346 [ $\mu \mathrm{g} / \mathrm{dl}]$, TRSF $16-50 \%$.

Transferrin saturation fraction (TRSF) was calculated from the following equation: $T R S F=\frac{\text { serum iron concentration }}{\text { TIBC }} 100 \%$.

CRP concentration.

Determining of CRP concentration was made with immunoturbidimetric TIA method with the used of reagents "Turbiquant CRP" by DADE BEHRING, Liederbach, Germany. Reference range of CRP concentration in serum is up to $0.6 \mathrm{mg} / \mathrm{dl}$.

\section{Erythrocyte parameters and indices}

Red blood cell count (RBC), hemoglobin $(\mathrm{Hb})$, hematocrit $(\mathrm{Ht})$, mean corpuscular volume of red blood cells (MCV), mean corpuscular hemoglobin $(\mathrm{MCH})$, mean corpuscular hemoglobin concentration (MCHC), red cell distribution width (RDW), were performed. For analysis of complete systemic blood Cell-Dyn 1700 apparatus by Abbott Diagnostics was used. Reference values: MCV [fl] 80-97, MCH [pg] 27-31.2, MCHC [g/dl] 31.8-35.4, RDW [\%] 11.6-14.8.

\section{Statistic analysis}

Statistic analyses were carried out with the use of Statistica 6.0 software. Normality of the distribution was checked with Shapiro-Wilk test. Comparative analysis of the parameters characterized with regular distribution was carried out with t-student test, and the remaining parameters, whose distribution was irregular, by non-parametric U Mann-Whitney test. In all tests the significance level was noted to be $<0.05$. 


\section{Results}

According to WHO, anemia is defined by hemoglobin $(\mathrm{Hb})$ level $<13 \mathrm{~g} / \mathrm{dL}$ for men and $<12 \mathrm{~g} / \mathrm{dL}$ for women (WHO, UNICEF, UNU. Iron deficiency anaemia, 1998). Non UC patients had severe anemia e.g. Hb level $<10 \mathrm{~g} / \mathrm{dL}$. None of the healthy controls had anemia.

On the basis of hemoglobin result anemia was found in all UC patients, 15 patients had iron deficiency anemia (IDA), 3 patients had anemia of chronic diseases (ACD), and in 2 patients both types of anemia coexisted (IDA/ACD). Patients with IDA as compared with control group had statistically significant decrease of hemoglobin levels $(p<0,001)$, red blood cell count $(\mathrm{RBC})$ count $(\mathrm{p}<0,01)$ and hematocrit levels $(\mathrm{p}<0,05)$. A significant decrease was also observed in serum iron level $(p<0,01)$, a case of ferritin concentration $(p<0.001)$. All patients with IDA had serum ferritin level below $100 \mu \mathrm{g} / \mathrm{L}$.

RDW ( $p<0,01)$ RDW was found to be statistically higher in UC patients with IDA than in control group. In contrast, other erythrocyte indices (MCV, $\mathrm{MCH}, \mathrm{MCHC}$ ), CRP concentration, unsaturated iron binding capacity (UIBC) and TIBC, were not statistically different in UC-IDA patients as compared in control. The results were presented in table 1.

In addition, in three patients diagnosed with ACD all patients had ferritin level above $100 \mu \mathrm{g} / \mathrm{L}$, and increased CRP. In addition RDW and TIBC/UIBC were in the normal range.

\section{Tests results of patients with IDA in moderately active stage of UC as compared with control group (C)}

\begin{tabular}{|l|c|c|c|}
\hline \multirow{2}{*}{} & IDA-UC & Control group & IDA-UC /C \\
\cline { 2 - 4 } & Mean \pm SD & Mean \pm SD & p \\
\hline Iron $[\mu \mathrm{g} / \mathrm{dl}]$ & $69,34 \pm 42,20$ & $92,8 \pm 25,4$ & $* 0,01$ \\
\hline Ferritin $[\mu \mathrm{g} / \mathrm{l}]$ & $56,1 \pm 68,0$ & $120,7 \pm 74,5$ & $* 0,001$ \\
\hline $\mathrm{Hb}[\mathrm{g} / \mathrm{dl}]$ & $11,5 \pm 3,9$ & $14,4 \pm 3,1$ & $* 0,001$ \\
\hline $\mathrm{RBC}[\mathrm{M} / \mu \mathrm{l}]$ & $4,2 \pm 0,22$ & $4,62 \pm 0,4$ & $* 0,01$ \\
\hline $\mathrm{Ht}[\%]$ & $34,7 \pm 5,7$ & $39,4 \pm 3,0$ & $* 0,05$ \\
\hline TIBC $[\mu \mathrm{g} / \mathrm{dl}]$ & $388,85 \pm 99,28$ & $333,0 \pm 75,12$ & 0,28 \\
\hline $\mathrm{UIBC}[\mu \mathrm{g} / \mathrm{dl}]$ & $239,61 \pm 101,12$ & $219,5 \pm 85,11$ & 0,84 \\
\hline TRFS $[\%]$ & $25,94 \pm 15,81$ & $33,13 \pm 12,08$ & 0,37 \\
\hline $\mathrm{MCV}[\mathrm{fl}]$ & $83,96 \pm 6,4$ & $87,64 \pm 3,56$ & 0,99 \\
\hline $\mathrm{MCH}[\mathrm{pg}]$ & $24,12 \pm 2,60$ & $29,2 \pm 2,61$ & 0,82 \\
\hline $\mathrm{MCHC}[\mathrm{g} / \mathrm{dl}]$ & $33,10 \pm 1,97$ & $34,05 \pm 1,09$ & 0,09 \\
\hline RDW $[\%]$ & $16,26 \pm 1,2$ & $13,16 \pm 0,82$ & $* 0,01$ \\
\hline $\mathrm{CRP}[\mathrm{mg} / \mathrm{dl}]$ & $0,72 \pm 0,52$ & $0,48 \pm 0,26$ & 0,22 \\
\hline
\end{tabular}

\section{Discussion}

Previous study large cohort of IBD patients have indicated that IDA was present in $53.7 \%$ and $\mathrm{ACD}$ in $12 \%$ of patients. In other report 44 outpatients with Crohn's disease and 55 with UC were stratified, and IDA was highly prevalent in patients with Crohn's disease (69.6\%) and ulcerative colitis (76.7\%), whereas ADA was shown 3\% of the patients with Crohn's disease and in 7\% of the patients with UC (Alves, Miszputen, Figueiredo, 2014). Importantly, anemia is more frequent in active disease (Oustamanolakis, 2011; Testa, 2016). 
In the present study we focused in most typical group of UC patients with moderate index of activity, and simultaneously diagnosed mild or moderate anemia. As noted in recent cohort study despite this, more than a third of UC patients are not tested for anemia in the time of diagnosis (Patel, 2018). Our aim is to provide a pathway to help achieve early diagnosis of type of anemia.

Pure IDA is present with serum ferritin $<30 \mu \mathrm{g} / \mathrm{L}$ and low hemoglobin levels (Stein, Dignass, 2013). Serum ferritin is an indicator of the storage of iron contents, however during inflammatory conditions such as UC, its serum concentration may be elevated like acute phase reactant (Umehara, Kudo, Nakaoka, Kawasaki, Shiomi, 2006). Thus if inflammation is present, the minimum cut-off level for ferritin when confirming IDA though to be $<100 \mu \mathrm{g} / 1$ (Stein, Dignass, 2013). In fact in our data all patients with IDA had ferritin serum level $<100 \mu \mathrm{g} / \mathrm{L}$. In three other anemic UC patients ferritin level was higher than $100 \mu \mathrm{g} / \mathrm{L}$, that in constellation with other iron pattern and increase CRP, indicate ACD. As compared with control group we found in patients with IDA significant decrease of ferritin level, but also increase of RDW value and TIBC and UIBC. Such constellation of iron patterns indicate IDA. The increase of TIBC is usually used for diagnosis of IDA in association with ferritin concentration. Wians et al. (Wians, Urban, Keffer, Kroft, 2001) shown in the group of IBD patients with IBD-IDA the average TIBC was $432 \pm 84 \mu \mathrm{g} / \mathrm{dl}$, as compared with significantly lower TIBC $-198 \pm 55 \mu \mathrm{g} / \mathrm{dl}$. in IBD-ADA patients. Lately single communication appeared (22 indicating the significance of red blood cell parameters in differentiation of anemia, especially in Crohn's disease. Some repots indicated that increased RDW which reflects the degree of anisocytosis, it could be considered as reliable marker in IDA (Oustamanolakis, 2011). Oustamanolakis et al (Oustamanolakis, 2011) suggested that RWD is one of the best markers for detecting IDA in patients with IBD. These authors have shown that RDW was significantly increased in IBD patients compared with control healthy subjects, and in IBD patients with IDA compared with non-anemic IBD patients, which tend to corroborate with our results. The number of cases of UC patients with ADA was too small to be statistically compared with IDA patients. Most researchers have found high perseverance IDA vs ACD in IBD patients. Pure ACD is mainly indicated by levels of serum ferritin > $100 \mu \mathrm{g} / \mathrm{L}$, and lower hemoglobin level, and elevated CRP (10). In addition TIBC is lower or within normal ranges, and RDW value is within referential ranges. Such constellation of examined parameters we have found to be diagnosed of ACD.

For example: the typical patient with IDA (W.G., man) had lower iron level $(44[\mu \mathrm{g} / \mathrm{dl})$ reduced ferritin level $(20,2 \mu \mathrm{g} / \mathrm{L})$ increased TIBC value $(463 \mu \mathrm{g} / \mathrm{dl})$. In addition, he had decreased MCV (79,7 fl), MCH (25,1 pg) MCHC (31,3 g/dl), and increased and RDW $(16,0 \%)$. CRP $(0,6 \mathrm{mg} / \mathrm{dl}$.) was in upper limit of normal value. In striking contrast, the typical patient with ACD (W.K, woman) had higher ferritin concentration $(113 \mu \mathrm{g} / \mathrm{L})$, and increased CRP level $(2,3 \mathrm{mg} / \mathrm{dl})$, whereas other iron pattern parameters $\mathrm{Fe}(64 \mu \mathrm{g} / \mathrm{dl})$ and TIBC $(357 \mu \mathrm{g} / \mathrm{dl})$ were in normal range. Similarly, erytrocytic indices: MCV $(88,4 \mathrm{fl})$ $\mathrm{MCH}(29,7 \mathrm{pg}), \mathrm{MCHC}(34,3 \mathrm{~g} / \mathrm{dl})$, and RDW (14,2\%) were within normal values.

It should be noted that in active IBD with increase CRP as acute phase protein inflammation is mediated by proinflammatory cytokines. IL-6 causes synthesis, and serum elevation of hepcidin. Hepcidin can block the intestinal iron absorption in the duodenum, and in addition it also causes reduced release of iron from iron storing cells, leading to functional iron deficiency, and in turn to ACD (Mecklenburg, 2014). Swiss IBD Cohort Study Group have presented data showing that serum hepcidin concentrations correlate with ferritin in patients 
with IBD (Mecklenburg, 2014). The differentiation between IBD and ADA is practically significant. In UC patients with moderate stage of IDA (as presented in our data) oral iron supplementation in doses $\leq 100 \mathrm{mg}$ /day would be recommended. If oral iron is not well tolerated, or this treatment is not sufficient intravenous iron preparation should be used (Lindgren, 2009; Lachance, 2011). However in ADA patients only iron given intravenously as first line treatment will bypass the hepcidin blockage.

\section{References}

Bernstein Ch.N., Benchimol E.I., Bitton A., Murthy S.K., MD, Nguyen G.C. et al. (2019). The Impact of Inflammatory Bowel Disease in Canada 2018: Extra-intestinal Diseases in IBD. J Can Assoc Gastroenterol (Suppl 1), S73-S80.

Stadnicki A., Colman R.W. (1998). Extraintestinal manifestations of inflammatory bowel disease: new insights into pathogenesis and clinical setting. Gastroenterol Pol 5, 517-523.

Lucendo A.J., Arias Á., Roncero Ó., Hervias D., Verdejo C. et al. (2017). Anemia at the time of diagnosis of inflammatory bowel disease: Prevalence and associated factors in adolescent and adult patients. Dig Liver Dis 49 (4), 405-411.

Oustamanolakis P., Koutroubakis I.E., Messaritakis I., Kefalogiannis G., Niniraki M., Kouroumalis E.A. (2011). Measurement of reticulocyte and red blood cell indices in the evaluation of anemia in inflammatory bowel disease. J Crohns Colitis 5 (4), 295-300.

Testa A., Rispo A., Romano M., Riegler G., Selvaggi F. et al. (2016). The burden of anaemia in patients with inflammatory bowel diseases. Dig Liver Dis 48 (3), 267-270.

Battat R, Kopylov U, Szilagyi A, Saxena A., Rosenblatt D.S. et al. (2014). Vitamin B12 deficiency in inflammatory bowel disease: prevalence, risk factors, evaluation, and management. Inflamm Bowel Dis 20 (6), 1120-8.

Clarke K., Sagunarthy R., Kansal S. (2008). RDW as an additional marker in inflammatory bowel disease/undifferentiated colitis. Dig Dis Sci 53, 2521-2523.

Gomollon F., Gisbert J.P. (2009). Anemia and inflammatory bowel diseases. World J Gastroenterol 15, 4659-65.

Bertero M.T., Caligaris-Cappio F. (1997). Anemia of chronic disorders in systemic autoimmune diseases. Haematologica 82, 375-81.

Weiss G., Goodnough L.T. (2005). Anemia of chronic disease. N Engl J Med 352, 1011-1023. Mecklenburg I., Reznik D., Fasler-Kan E., Drewe J., Beglinger C., Hruz P. (2014). Swiss IBD Cohort Study Group. Serum hepcidin concentrations correlate with ferritin in patients with inflammatory bowel diseases. J Chrn's Colitis 8 (11), 1392.

Vermeire S., Van Assche G., Rutgeerts P. (2006). Laboratory markers in IBD: useful, magic, or unnecessary toys? Gut., 55, 426-31.

Anker S.D., Comin Colet J., Filippatos G., et al. (2009). Ferric carboxymaltose in patients with heart failure and iron deficiency. $N$ Engl J Med 361, 2436-2448.

Shepshelovich D., Zvi B.R., Avni T., Gafter U., Gvili G.A. (2016). Intravenous versus oral iron supplementation for the treatment of anemia in $C K D$ : an updated systematic review and metaanalysis. Am J Kidney Dis 68, 677-690.

Truelove S.C., Witts L.J. (1955). Cortisone in ulcerative colitis; final report on a therapeutic trial. Br Med J 2, 1041-1048.

WHO, UNICEF, UNU. Iron deficiency anaemia: Assessment, prevention and control. Report of a joint WHO/UNICEF/UNU consultation. Geneva: World Health Organization 1998. 
Alves R.A., Miszputen S.J., Figueiredo M.S. (2014). Anemia in Inflammatory Bowel Disease: Prevalence, Differential Diagnosis and Association With Clinical and Laboratory Variables Sao Paulo Med J 132 (3), 140-6.

Patel D., Trivedi C., Khan N. (2018). Management of Anemia in Patients with Inflammatory Bowel Disease (IBD). Curr Treat Option Gastroenterol 16 (1), 112-128.

Stein J., Dignass A.U. (2013). Management of iron deficiency anemia in inflammatory bowel disease - a pratical approach. Ann Gastroenterol 26 (2), 104-113.

Umehara Y., Kudo M., Nakaoka R., Kawasaki T., Shiomi M. (2006). Serum proinflammatory cytokines and adhesion molecules in ulcerative colitis. Hepatogastroenterology 53, 879-882.

Wians F.H., Urban J.E., Keffer J.H., Kroft S.H. (2001). Discriminating between iron deficiency anemia and anemia of chronic disease using traditional indices of iron status vs transferrin receptor concentration. Am J Clin Pathol. 115 (1), 112-118.

Molnar T., Farkas K., Szepes Z., Nagy F., Nyari T., Wittmann T. (2008). RDW can be a useful additional marker in diagnosing Crohn's disease and ulcerative colitis. Dig Dis Sci 53, 2521-3. Lindgren S., Wikman O., Befrits R. et al (2009). Intravenous iron sucrose is superior to oral iron sulphate for correcting anaemia and restoring iron stores in IBD patients: A randomized, controlled, evaluator-blind, multicentre study. Scand J Gastroenterol 44, 838-845.

Lachance K., Savoie M., Bernard M. et al. (2011). Oral ferrous sulfate does not increase preoperative hemoglobin in patients scheduled for hip or knee arthroplasty. Ann Pharmacother 45, 764-770. 